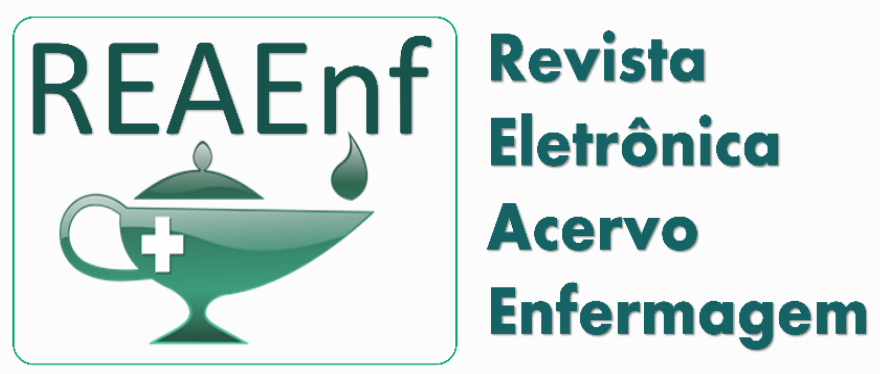

RELATO DE EXPERIÊNCIA

Recebido em: $8 / 2020$

Aceito em: 9/2020

Publicado em: 12/2020

\title{
Arteterapia como opção não medicamentosa para pacientes internados em uma unidade hospitalar
}

Artetherapy as a non-drug option for patients included in a hospital unit

La arteterapia como opción sin fármacos para pacientes incluidos en unidad hospitalaria

Silvana Carloto Andres ${ }^{1 \star}$, Rodrigo Dornelas ${ }^{1}$, Patrícia Bitencourt Toscani Greco ${ }^{2}$, Rafaella França Torres $^{3}$, Vera Rocha ${ }^{4}$.

Resumo: Relatar a vivência de uma atividade de arteterapia realizada com pacientes internados em uma unidade de clínica medica em um hospital de pequeno porte do interior do estado do Rio Grande do Sul. Trata-se de um relato de experiência sobre a prática da arteterapia aplicada a pacientes adultos internados, a atividade ocorreu no período de setembro a outubro de 2019. Foram convidados a participar 18 pacientes adultos internados em uma unidade de clínica médica adulta. As vivências da Arteterapia objetivaram proporcionar bem-estar, diminuir o estresse e a ansiedade, tornando o processo de internação mais leve e humanizado, bem como, buscando elevar a autoestima e promover autoconhecimento dos pacientes internados. O processo de Arteterapia é uma intervenção de baixo custo, não farmacológica além de ter uma importante influência na mudança de hábitos, qualidade de vida dos pacientes envolvidos, pode ser associado a terapia medicamentosa melhorando a sintomatologia e patologia do paciente.

Palavras-chave: Terapias complementares, Hospitalização, Enfermagem.

\begin{abstract}
To report the experience of an art therapy activity performed with patients admitted to a medical clinic unit in a small hospital in the interior of the state of Rio Grande do Sul. This is an experience report on the practice of applied art therapy. for adult hospitalized patients, the activity took place from September to October 2019. Eighteen adult patients admitted to an adult medical clinic unit were invited to participate. The experiences of Art Therapy aimed to provide well-being, reduce stress and anxiety, making the hospitalization process lighter and more humanized, as well as seeking to raise self-esteem and promote self-knowledge of hospitalized patients. The Art Therapy process is a low-cost, non-pharmacological intervention, in addition to having an important influence on changing habits, the quality of life of the patients involved, it can be associated with drug therapy, improving the patient's symptoms and pathology.
\end{abstract}

Keywords: Complementary therapies, Hospitalization, Nursing.

\footnotetext{
${ }_{1}^{1}$ Fundação Oswaldo Cruz (Fiocruz), Rio de Janeiro - RJ. *E-mail: silvana.andres@yahoo.com.br

2 Universidade Regional Integrada do Alto Uruguai e das Missões, Santiago - RS.

3 Universidade Federal de Santa Maria, Palmeira das Missões - RS.

${ }^{4}$ Universidade Federal do Rio Grande do Sul. Porto Alegre - RS.
} 
Resumen: Relatar la experiencia de una actividad de arteterapia realizada con pacientes ingresados en una unidad clínica médica de un pequeño hospital del interior del estado de Rio Grande do Sul. Se trata de un relato de experiencia sobre la práctica de arteterapia aplicada. para pacientes adultos hospitalizados, la actividad se llevó a cabo de septiembre a octubre de 2019. Se invitó a participar a dieciocho pacientes adultos ingresados en una unidad de clínica médica para adultos. Las experiencias de Arteterapia tuvieron como objetivo brindar bienestar, reducir el estrés y la ansiedad, hacer más liviano y humanizado el proceso de hospitalización, así como buscar elevar la autoestima y promover el autoconocimiento de los pacientes hospitalizados. El proceso de Arteterapia es una intervención no farmacológica de bajo costo, además de tener una influencia importante en el cambio de hábitos, la calidad de vida de los pacientes involucrados, puede asociarse a la farmacoterapia, mejorando los síntomas y patología del paciente.

Palabras Clave: Terapias complementarias, Hospitalización, Enfermería.

\section{INTRODUÇÃO}

A hospitalização é um momento delicado para o paciente, onde ela se ausenta de suas atividades cotidianas, tendo que enfrentar a dura realidade de um hospital, que muitas vezes não tem um ambiente agradável e acolhedor para receber essa clientela (OLIVEIRA SR e ALMEIDA NA, 2016). Sendo assim o processo de hospitalização se torna marcado por insegurança e desconforto, combinado de anseios e medos por estar rodeada de pessoas desconhecidas em ambiente hostil (GONÇALVES KT, et al., 2017). Essa alteração na rotina desses indivíduos pode tornar-se menos traumática, se algumas técnicas não medicamentosas, voltadas para um atendimento humanizado seja empregado pela equipe de saúde.

Uma das medidas não farmacológicas, que pode ser associada a outros métodos medicamentosos, para amenizar o processo da internação hospitalar, pode ser a arteterapia. Essa técnica pode ser empregada em treinamentos empresariais, reabilitação psicossocial, projetos comunitários de educação e para a saúde, em consultórios e clínicas especializadas com doenças generativas e outros (CUNHA EFB, et al., 2017). A prática da arteterapia, é conhecida como um dispositivo terapêutico que restabelece de forma não verbal, visando a resgatar o autoconhecimento e transformação, procurando aceitar os diversos aspectos dos pacientes, como os afetivos, culturais, cognitivos, motores, sociais entre outros, a arteterapia engloba os saberes de diversas áreas, constituindo-se como uma prática transdisciplinar. (DOS REIS, 2014).

Em 2006 o governo federal criou a Política Nacional de Práticas Integrativas e Complementares (PNPIC), Portaria 971 GM/MS de 3 de maio de 2006, que trouxe diretrizes norteadoras para Medicina Tradicional Chinesa/Acupuntura, Homeopatia, Plantas Medicinais e Fitoterapia, Medicina Antroposófica e Termalismo Social/Crenoterapia, e em 2017 a PNPIC inclui a Arteterapia, e mais 12 práticas, para serem desenvolvidas nos Sistema Único de Saúde do Brasil (SUS), e em 2018 o Ministério da Saúde amplia novamente a PNPIC, ofertando atualmente 29 práticas integrativas e complementares em âmbito nacional nas unidades SUS (BRASIL, 2018).

Sendo assim, as Práticas Complementares e integrativas em Saúde (PICS) contribuem para a busca da integralidade da atenção à saúde e para redução dos gastos públicos, uma vez que as PICS possibilitam o complemento do tratamento medicamentoso tradicional/usual de forma simples e de baixo custo, reduzindo assim o processo de internação e/ou o uso de medicamentos (TELESI JE, 2016).

A arteterapia é um recurso terapêutico utilizado por diversas instituições para o tratamento de pessoas que possuem algum transtorno mental e de reabilitação física (DOS REIS, 2014). Trata-se, portanto, de uma prática terapêutica que tem possibilitado diferentes formas de acesso à saúde física e mental, ao bemestar, à reintegração e inclusão social dos pacientes e à melhoria de sua qualidade de vida.

A arteterapia é uma técnica que tem suas próprias teorias sendo aplicadas de forma multiprofissional abrangendo enfermeiros, assistentes sociais, fisioterapeutas, psicólogos, médicos em diferentes cenários, utilizando a prática como uma grande diversidade de recursos que expressam elementos terapêuticos e desenvolvimento pessoal (MOREIRA MAG e SANTOS VC, 2019). O profissional enfermeiro, é o profissional 
que mais mantem cuidado com o paciente, por isso, nesse cenário, assumindo assim a condição de apropriação de algumas práticas complementares integrativas/terapêuticas não farmacológicas e as coloque-as em prática complementando o cuidado do paciente, cuidado esse objeto de trabalho da enfermagem (ALMEIDA JR, et al., 2019). Sendo assim, possível que o enfermeiro visualize o paciente como um todo.

O trabalho relata a experiência desenvolvida utilizando a Arteterapia, sendo essa uma das terapias reconhecidas na Portaria 849/2017, a atividade foi realizada com pacientes internados na unidade de clínica médica em um Hospital filantrópico de médio porte no interior do estado do Rio Grande do Sul.

\section{RELATO DE EXPERIÊNCIA}

Participaram da vivência em arteterapia de forma voluntária 18 pacientes adultos internados na clínica médica em uma unidade do SUS, em um hospital no interior do estado do Rio Grande do Sul. Dos participantes dez eram do sexo feminino e oito sexo masculino, a idade ficou entre 32 a 65 anos. As patologias dos internados eram doenças crônicas não transmissíveis (DCNT), essas doenças trazem prejuízos sociais, perda da qualidade de vida, limitações as pessoas em suas atividades cotidianas e ao isolamento social (COUTINHO ATQ, et al., 2018). Prevaleceram nesse contexto a hipertensão e diabetes melitus. A média de dias de internação ficou entre 18 a 36 dias. A vivência ocorreu nos meses de setembro e outubro de 2019.

Foram realizadas três sessões de arteterapia com duração de uma hora e meia cada, com as seguintes etapas, primeiro momento: apresentação da proposta de atividade, seguida de algumas perguntas que demonstrassem o entendimento da temática, buscando compreender a utilidade da arte. Segundo momento: explicações de como seria realizado e os materiais disponíveis para utilização, desenvolvimento da técnica de arteterapia. Terceiro momento: realização de mais uma oficina em arterapia e fechamento com a partilha das experiências.

Os três momentos foram realizados no decorrer de uma semana, intercalados por um dia de atividade, proporcionando assim que os mesmos pacientes participassem das atividades. Podendo obter melhor a observação e a experiência ser mais concreta.

Durante as sessões, utilizou-se um fundo musical para auxiliar na concentração e relaxamento. As figuras utilizadas para pintura foram de mandalas, para desenvolver as atividades, foram utilizadas materiais como tinta para papel, giz de cera, canetas coloridas, lápis de cor, pedaços de tecidos e papeis coloridos (como revistas) jornais e desenhos impressos em folhas de oficio tamanho A4.

Indicasse utilizar a arteterapia associada com a à técnica de colagem, utilizando revistas, para mobilizar conteúdos internos, lembrar emoções e despertar sentimentos, pois, as imagens permitem que a pessoa manifeste seu inconsciente (SILVA MEB, et al., 2018). Por esse motivo, utilizou-se revistas e materiais semelhantes, para recortes podendo deixar os pacientes livres na criação de suas artes.

As vivências da Arteterapia buscam melhorar a autoestima e promover/gerar autoconhecimento e percepção sobre o processo de saúde/doença, tornando o processo de internação menos traumatizante e estressante. Dos 18 pacientes, 12 deles solicitaram realizar mais de uma pintura de mandala, pois informando que gostaria de levar a pintura para casa.

Percebeu nesta vivência que os pacientes iniciavam o processo de arteterapia de forma individual, e após organizavam-se em grupos de forma espontânea. Nesse cenário da vivência, vários pacientes relataram estar felizes de ter uma atividade para realizar durante o processo de internação, e que realizando a atividade, sentiam-se mais felizes.

No último encontro foi possível partilhar os resultados, através de relatos das falas dos pacientes durante o processo de participação das experiências vivenciadas pelas oficinas de arteterapia, e compartilhados entre eles através de relatos verbais, escritos ou pintados. Após o encerramento dos momentos pode-se elencar duas categorias, que foram mais percebidas na vivência.

REAEnf/EJNC | Vol. 7 | e4843 | DOI: https://doi.org/10.25248/REAenf.e4843.2020 Página 3 de 6 


\section{DISCUSSÃO}

A arteterapia cada vez mais abrange espaço na área da saúde, pois essas ferramentas fazem com que os profissionais possam ajudar seus pacientes de maneira mais leve no processo de hospitalização. $O$ trabalho multiprofissional é importante nessa terapia, já que a ideia não é apenas propor uma atividade e sim planejar e reforçar as relações interpessoais, fazendo com que se crie um vínculo entre paciente e profissional, atuando na melhora da condição de saúde do paciente (VITAL TS, et al., 2020).

Todos os seres humanos precisam desenvolver-se, seria interessante experimentar a arteterapia, sendo que ela visa não apenas o momento e sim o processo como um todo. Podendo auxiliar no processo de autoconhecimento e reconhecimento como um todo da cultura da qual o individuo está inserido. A arte desenvolvida deve ser usado como uma fonte de comunicação. De um modo geral, a terapia pode auxiliar também na interação social, fazendo a inclusão de diversas pessoas (ANASTASIOU HP e MATOS PJS, 2020). Sendo assim elencou-se como itens para discussão: O sentimento e benefícios proporcionados pela arteterapia; O processo de hospitalização e o uso da arteterapia desenvolvido pela enfermagem.

\section{Os sentimentos e benefícios proporcionados pela arteterapia}

Várias experiências com a utilização da Arteterapia com pacientes, vem demostrando benefícios emocionais, físicos e psicológicos, e vem apresentando crescimento dessa prática com pacientes em todos os níveis de atenção à saúde, essas práticas tem sido relatadas e apontam de forma cientifica para a consolidação do seu uso (SILVA MEB, et al., 2018).

A Arterapia tem sido utilizada como prática do cuidado em saúde, através do uso das expressões artísticas como forma terapêutica, os benefícios têm estimulado a utilização da arte no processo psicoterapêutico, para atendimento de pacientes com patologias crônicas e agudas (FACCO SCM, et al., 2016).

A arteterapia pode ser aplicada a indivíduos ou grupos, em variados locais e contextos, e utiliza a expressão artística como processo terapêutico, sendo capaz de promover a melhoria da qualidade de vida, o desenvolvimento pessoal e contribuir com a saúde mental, que é definida como um estado de bem-estar em que cada indivíduo percebe seu próprio potencial e consegue lidar com as tensões normais da vida (BERNARDO PP, 2013).

Utilizando as várias linguagens artísticas, os pacientes poderão expressar pensamentos, emoções e sentimentos, recuperação e conquista de bem-estar e de melhoria da qualidade de vida (FACCO SCM, et al., 2016). O exercício cerebral implícito à ação de criar, utilizando as várias possibilidades de linguagens, promove a melhoria das condições física, afetiva e mental do indivíduo, levando-o à obtenção da harmonia do todo (BERNARDO PP, 2013).

Portanto a arteterapia é uma prática, que atua de forma dinâmica canalizando, as variáveis do adoecimento mental e físico de forma positiva, havendo assim uma diminuição dos fatores negativos como os psicológicos e os físicos, que muitas vezes, surgem como sintomas associados as doenças/patologias (BARRETO JR, et al., 2011).

Podendo ser aplicada a pacientes de todas as faixas etárias, ajudado o paciente a reabilitação física e mental, com maior espaço maior no campo mental (FACCO SCM, et al., 2016). É uma prática utilizada para prevenção, promoção e reabilitação do indivíduo (BRASIL, 2018).

Desta forma, uma oficina de arteterapia não funciona como tratamento médico para nenhum tipo de patologia ou mal, mas como uma prática alternativa não medicamentosa, de valorizar o ser humano e de proporcionar bem-estar.

\section{O processo de hospitalização e o uso da arteterapia desenvolvido pela enfermagem}

Entre os profissionais de saúde, os enfermeiros são os que mantêm maior contato com os pacientes no que tange o cuidado dentro dos serviços de saúde, tendo grande potencial para detectar os problemas ou agravos de saúde. As práticas integrativas e complementares têm sua inserção ainda recente no contexto

REAEnf/EJNC | Vol. 7 | e4843 | DOI: https://doi.org/10.25248/REAenf.e4843.2020 Página 4 de 6 
de saúde brasileiro. Neste caso, a enfermagem dispõe de um papel fundamental no que se refere ao conhecimento, acesso e aplicabilidade dessas práticas reconhecidas e respaldadas pela legislação vigente para atuarem em locais públicos ou privados (ANDRES FC, 2020).

O Conselho Federal de Enfermagem (COFEN), apoia a iniciativa do Ministério da Saúde com as práticas complementares na Política Nacional de Práticas Integrativas e Complementares no Sistema Único de Saúde (PNPIC) e destaca que é uma evolução na área de saúde e na prevenção e promoção do cuidado com ênfase no grande número de profissionais da Enfermagem neste campo, podendo atuar de forma regular de acordo com as políticas nacionais existentes (COFEN, 2018).

Essas práticas, em especial a arteterapia, busca estimular mecanismos naturais de prevenção de agravos e promoção da saúde por meio de tecnologias eficazes, seguras e por vezes de baixo custo, com base na escuta acolhedora, no desenvolvimento do vínculo terapêutico e na integração do ser humano.

A hospitalização e o adoecimento são processos que podem viabilizar a produção de sofrimento no sujeito. Lidar com os limites do corpo e dor física diante da nova realidade suscita o encadeamento de questões emocionais até então não vivenciadas (LIMA KYN, 2014).

Os resultados mostraram que o processo de Arteterapia é uma intervenção de baixo custo, não farmacológica. Com base nos inúmeros benefícios descritos, revisão de literatura sugere que programas com artes sejam inseridos em ambientes de cuidado à saúde, inclusive pela característica holística, baixo custo e o potencial de promoção de saúde mental pública e bem-estar a todos os envolvidos (JENSEN A, BONDE LO, 2018). A arteterapia pode ser desenvolvida em outros espaços, com outros públicos, pois transcorre o sentido da arte, ela traz a promoção da saúde em meio a esses sentidos.

A Arteterapia tem sido aplicada a pessoas com diferentes doenças e contextos para identificar o estado emocional dos seus participantes e ajudar na remissão de sintomas depressivos, físicos o que auxilia no processo de reabilitação e redução no processo de internação influenciando na redução de gastos públicos e melhora no bem-estar do paciente (ZUBALA A, et al., 2014).

Avaliando este trabalho, destaca-se a dificuldade em sua realização, como a produção e publicação escassa de estudos relacionados à temática da arteterapia, desenvolvidas em hospitais com adultos. Essa limitação, assim como, o desenvolvimento deste estudo, sugere novas pesquisas relacionadas a essa temática, as quais favoreçam a promoção da saúde. Assim, diante do conjunto de evidências geradas na prática, é de grande necessidade a arteterapia como método terapêutico amenizando as tensões do processo de internação promovendo bem-estar dos pacientes, destacando a importância da inserção do profissional enfermeiro nestes espaços de cuidado, gerando a prevenção e reabilitação da saúde.

\section{REFERÊNCIAS}

1. ALMEIDA JR, et al. O enfermeiro frente ás práticas integrativas e complementares em saúde na estratégia de saúde da família. Revista Eletrônica Acervo Saúde. 2019; (18), 71 -77.

2. ANASTASIOU HP, MATOS PJS. Arteterapia: Considerações ao processo. Revista Integrativa em Inovações Tecnológicas Nas Ciências da Saúde. 2020; 3(00), 61-78.

3. BARRETO JR, et al. Arteterapia humanização em saúde: uma prática no tratamento de idosos com vestibulopatias. Revista Equilíbrio Corporal e Saúde, 2011;3(2):45-53.

4. BERNARDO PP. A prática da arteterapia: correlações entre temas e recursos. Temas centrais em arteterapia. $4^{\mathrm{a}}$ ed. v. 1. São Paulo: Arterapinna Editorial; 2013.

5. BRASIL. Ministério da Saúde. Portaria no 702, de 21 de março de 2018. Altera a Portaria de Consolidação $n^{\circ}$ 2/GM/MS, de 28 de setembro de 2017, para incluir novas práticas na Política Nacional de Práticas Integrativas e Complementares - PNPIC, Brasília, 2018.

6. COFEN. Conselho Federal de Enfermagem. Manifesta apoio às Práticas Integrativas e Complementares. Brasília, Cofen 2018. Disponível em: http://www.cofen.gov.br/cofenmanifesta-apoio-as-praticas-integrativas-ecomplementares_61201.html. Acesso em: 10 mar 2020.

7. COUTINHO ATQ, et al. Comunicação social e independência funcional em idosos de comunidade coberta pela estratégia saúde da família. Revista CEFAC. 2018; 20(3): 363-373. 
8. CUNHA EFB, et al. Arteterapia Na Perspectiva Junguiana: Expressão Psíquica E Transformação Do Ser. Revista Científica Univiçosa - Volume 9- n. 1 - Viçosa-MG 2017.

9. DOS REIS AC. Arteterapia: a Arte como Instrumento no Trabalho do Psicólogo. PSICOLOGIA: CIÊNCIA E PROFISSÃO. 2014; 34 (1), 142-157.

10. FACCO SCM, et al. A arteterapia no tratamento dos usuários de um centro de atenção psicossocial. Revista Espaço Ciência \& Saúde. 2016; (4):45-54.

11. GONÇALVES KT, et al. Criança Hospitalizada E Equipe De Enfermagem: Opinião De Acompanhantes. Rev enferm UFPE on line., Recife. 2017;11(6):2586-2593.

12. JENSEN A, BONDE LO. The use of arts interventions for mental health and wellbeing in health settings. Perspect Public Health [Internet]. 2018;138 (4):209-214.

13. LIMA KYN, et al. Atividade lúdica como ferramenta para o cuidado de enfermagem às crianças hospitalizadas. REME. Revista Mineira de Enfermagem.2014; 18 (3):741-746.

14. MOREIRA MAG, SANTOS VC. A fotografia como arteterapia no processo de recuperação de mulheres após o tratamento de câncer de mama. Orientador: Virgínia Rozendo de Brito. 2019. 16f. Trabalho de Conclusão de Curso (Bacharel em Enfermagem) - Centro Universitário do Planalto Central Aparecido dos Santos. Distrito Federal, 2019.

15. OLIVEIRA, SR, ALMEIDA NA. Utilizando as expressões lúdicas como terapêutica na reabilitação da hospitalização infantil uma revisão integrativa. Revista Eletrônica Gestão \& Saúde.2016; 07 (01): 356-68.

16. TELESI JE. Práticas integrativas e complementares em saúde, uma nova eficácia para o SUS. Estudos Avançados. São Paulo. 2016; 30 (86): 99-112.

17. VITAL TS, et al. Relações entre arteterapia em crianças hospitalizadas com câncer. Revista Diálogos Interdisciplinares. 2020; 9(4): 75-83.

18. ZUBALA A, et al. Prática de psicoterapia artística com adultos que sofrem de depressão no Reino Unido: resultados qualitativos de um questionário específico para depressão. As Artes em Psicoterapia. 2014; 41 (5): 563-569. 